\title{
Mesoscale hydrodynamic simulation of short polyelectrolytes in electric fields
}

Sandra Frank, and Roland G. Winkler

Citation: The Journal of Chemical Physics 131, 234905 (2009); doi: 10.1063/1.3274681

View online: https://doi.org/10.1063/1.3274681

View Table of Contents: http://aip.scitation.org/toc/jcp/131/23

Published by the American Institute of Physics

\section{Articles you may be interested in}

Effect of hydrodynamic correlations on the dynamics of polymers in dilute solution

The Journal of Chemical Physics 138, 144902 (2013); 10.1063/1.4799877

Multiparticle collision dynamics simulations of viscoelastic fluids: Shear-thinning Gaussian dumbbells The Journal of Chemical Physics 138, 104903 (2013); 10.1063/1.4792196

Mesoscale hydrodynamic modeling of a colloid in shear-thinning viscoelastic fluids under shear flow The Journal of Chemical Physics 135, 134116 (2011); 10.1063/1.3646307

Conformational and rheological properties of semiflexible polymers in shear flow

The Journal of Chemical Physics 133, 164905 (2010); 10.1063/1.3497642

Polyelectrolyte solutions with added salt: A simulation study

The Journal of Chemical Physics 119, 1813 (2003); 10.1063/1.1580109

Hydrodynamic correlations and diffusion coefficient of star polymers in solution

The Journal of Chemical Physics 141, 084901 (2014); 10.1063/1.4893766

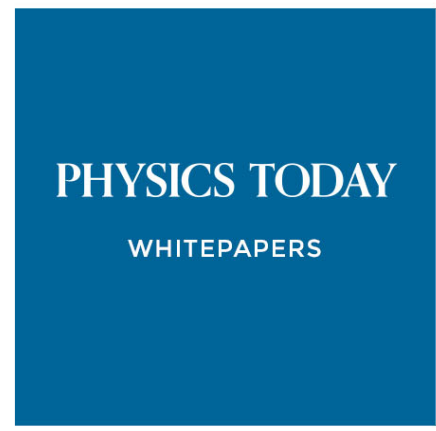

ADVANCED LIGHT CURE ADHESIVES

Take a closer look at what these environmentally friendly adhesive systems can do

\section{READ NOW}

PRESENTED BY

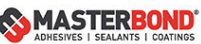




\title{
Mesoscale hydrodynamic simulation of short polyelectrolytes in electric fields
}

\author{
Sandra Frank ${ }^{1, a)}$ and Roland G. Winkler ${ }^{2, b)}$ \\ ${ }^{1}$ Institut für Theoretische Physik, Georg-August-Universität, 37077 Göttingen, Germany \\ ${ }^{2}$ Institut für Festkörperforschung, Forschungszentrum Jülich, 52425 Jülich, Germany
}

(Received 29 September 2009; accepted 24 November 2009; published online 18 December 2009)

\begin{abstract}
The dynamical, conformational, and transport properties of short flexible polyelectrolytes are studied in salt-free solution under the influence of an external electric field taking hydrodynamic interactions into account. A coarse-grained polymer model is applied and the multiparticle collision dynamics approach is adopted to account for the solvent. We consider various Coulomb interaction strengths and polymer lengths. The scaling behavior of the diffusion coefficient with respect to polymer length strongly depends on the charge interaction strength due to changes in the polyelectrolyte conformations by counterion condensation. The diffusion coefficients at weak Coulomb interaction strengths can well be described by the expression of rodlike objects, whereas at stronger electrostatic interactions, we observe a Zimm-like behavior. The polyelectrolyte mobilities obey the Nernst-Einstein relation at weak charge interactions. For large interaction strengths and long polymers, the mobilities are independent of molecular weight because of the screening of the intramolecular Coulomb interactions by counterions. Our studies demonstrated that the dynamics of polyelectrolytes in dilute solution and in an external field is governed by an interplay of hydrodynamic interactions and counterion condensation. (C) 2009 American Institute of Physics. [doi:10.1063/1.3274681]
\end{abstract}

\section{INTRODUCTION}

Polyelectrolytes play a major role in biology and engineering and are of considerable interest for soft matter physics from a basic scientific point of view. The long-range Coulomb interactions between the charged components (monomers, counterions, and coions) of the polyelectrolyte solution are the origin of extraordinary dynamical and conformational properties, which deviate strongly from those of neutral polymers. ${ }^{1-11}$

Naturally, these specificities will also strongly influence the behavior of polyelectrolytes in an external electric field. Since electrophoresis is a standard tool in technical applications to separate charged objects according to molecular weight, an understanding of the underlying physical mechanisms is of fundamental importance for the development and design of novel separation techniques. In electrophoresis, the polyelectrolyte chains and ions in the solution respond to the applied electric field with a drift motion. Their transport and conformational properties are determined by the electric field, ${ }^{12,13}$ effects induced by Coulomb interactions, such as counterion condensation and charge screening, as well as hydrodynamic interactions. Because of the complexity of the problem, it is not surprising that it is not at all clear to what extent these different aspects influence the dynamical and structural behavior of the system's constituents.

Experimentally, the electrophoretic mobility has been studied extensively (cf. Refs. 14-18 and references therein). In particular, it has been observed that the mobility is inde-

\footnotetext{
${ }^{a)}$ Electronic mail: sandra.frank@ phys.uni-goettingen.de.

${ }^{b)}$ Electronic mail: r.winkler@fz-juelich.de.
}

pendent of molecular weight above a certain threshold. ${ }^{14-17}$ This has also been explained in theoretical approaches. ${ }^{13,14,19-21}$ Recently, a surprising behavior was found for shorter chains of synthetic polyelectrolytes such as polystyrene sulfonate (PSS). ${ }^{15,16,18}$ The mobility first increases for very short chains, reaches a maximum, and finally decreases again and approaches a constant value for long polymers. This nonmonotonic behavior has also been observed in recent computer simulation studies. ${ }^{22,23}$ It turned out that such a behavior seems not to be specific to synthetic polyelectrolytes only, since similar results have been reported for single-stranded DNA. ${ }^{24}$

Full insight into the interplay of the various interactions present in free flow electrophoresis is difficult to achieve by analytical theory, particularly due to the nonequilibrium and nonlinear character of the problem. Computer simulations, specifically mesoscale simulations which take hydrodynamic interactions adequately into account, provide the opportunity to tackle the challenging problem and to arrive at a molecular understanding. ${ }^{22,23}$

In this article, we present results of a mesoscale hydrodynamics simulation study of the dynamical and conformational properties of dilute polyelectrolyte solutions exposed to external electric fields. Hydrodynamic interactions are explicitly taken into account by the multiparticle collision dynamics (MPC) algorithm. ${ }^{25-29}$ Our purpose is, on the one hand, to elucidate the influence of the Coulomb interaction strength and, on the other hand, to clarify the influence of hydrodynamic interactions and counterion condensation on the dynamics of a polyelectrolyte molecule. Hence, we will present results for the mobilities of the chain molecules as 
well as the mobilities of its condensed and uncondensed counterions for polyelectrolytes of various lengths and Coulomb interaction strengths. In addition, we calculate the number of condensed counterions, diffusion coefficients, and discuss the conformational properties of the polyelectrolytes. Moreover, field induced charge asymmetries are characterized in terms of dipole moments and the spatial distribution of counterions.

The paper is organized as follows: In Sec. II, the polyelectrolyte model is introduced and in Sec. III the simulation method is outlined. The model parameters are summarized in Sec. IV. Section V presents the results and Sec. VI summarizes our findings.

\section{MODEL}

The linear polyelectrolyte molecule consists of $N_{m}$ monomers each carrying a negative elementary charge $(-e)$. Subsequent monomers are connected by the harmonic bond potential

$$
U_{B}=\frac{\kappa}{2} \sum_{i=1}^{N_{m}-1}\left(\left|\boldsymbol{r}_{i+1}-\boldsymbol{r}_{i}\right|-l_{b}\right)^{2}
$$

with $\boldsymbol{r}_{i}$ the position of particle $i$, the bond parameter $l_{b}$, and the force constant $\kappa$. The chain is surrounded by $N_{m}$ positively charged $(+e)$ monomers, which represent the counterions such that the total system is electrically neutral. In order to prevent the monomers from penetrating each other, pairwise excluded volume interactions are taken into account by the potential $U_{\mathrm{LJ}}=\sum_{i=1}^{2 N_{m}-1} \sum_{j=i+1}^{2 N_{m}} u_{\mathrm{LJ}}\left(r_{i j}\right)$, where $r_{i j}=\left|\boldsymbol{r}_{i}-\boldsymbol{r}_{j}\right|$ is the distance between the monomer $i$ and $j$ and $u_{\mathrm{LJ}}\left(r_{i j}\right)$ is the truncated and shifted Lennard-Jones potential ${ }^{5,30}$

$$
u_{\mathrm{LJ}}\left(r_{i j}\right)=\left\{\begin{array}{ll}
4 \epsilon\left[\left(\frac{\sigma}{r_{i j}}\right)^{12}-\left(\frac{\sigma}{r_{i j}}\right)^{6}\right]+\epsilon, & r_{i j}<2^{1 / 6} \sigma \\
0, & \text { else }
\end{array} .\right.
$$

The long-range Coulomb interaction

$$
U_{C}=\frac{1}{4 \pi \varepsilon \varepsilon_{0}} \sum_{i} \sum_{j>i} \frac{q_{i} q_{j}}{\left|\boldsymbol{r}_{i}-\boldsymbol{r}_{j}\right|},
$$

where $\epsilon$ is the dielectric constant of the solvent and $q_{i}$ denotes the charge of a monomer or counterion, respectively, is treated by Ewald summation. ${ }^{31}$

The system is exposed to a homogeneous, constant external electric field $\boldsymbol{E}$ with the potential

$$
U_{E}=\sum_{i=1}^{2 N_{m}} q_{i} \boldsymbol{E r}_{i}
$$

\section{SIMULATION METHOD}

An important aspect in dilute soft matter solutions are hydrodynamic interactions mediated by the solvent. Since the simulation of large systems with all of its microscopic details of the involved molecules would overburden computational power by far, several mesoscale simulation methods have been developed to overcome this problem. An overview of the most common techniques can be found in Refs. 28, 29, and 32 .

We will apply the MPC algorithm. ${ }^{25-30}$ In the MPC method, the solvent is represented by a system of $N$ point particles at positions $\boldsymbol{r}_{i}(i=1, \ldots, N)$, velocities $\boldsymbol{v}_{i}$, and mass $m$ per particle in a cubic simulation box of side length $L$. Periodic boundary conditions are applied in all spatial directions. The MPC algorithm consists of streaming and collision steps. In the streaming step, the particles move freely during a time interval $h$, which is denoted as collision time. Hence, the particle positions are updated according to

$$
\boldsymbol{r}_{i}(t+h)=\boldsymbol{r}_{i}(t)+h \boldsymbol{v}_{i}(t) .
$$

In the collision step, the particles interact with each other by a stochastic process. For this purpose, the simulation box is partitioned into cubic collision cells of side length $a$ and the relative velocities of the particles in such a cell-with respect to the center-of-mass velocity of all the particles within the cell-are rotated by a fixed angle $\alpha$ around a random axis. The new velocities are then given by

$$
\boldsymbol{v}_{i}(t+h)=\boldsymbol{v}_{\mathrm{cm}}(t)+\mathcal{R}(\alpha)\left[\boldsymbol{v}_{i}(t)-\boldsymbol{v}_{\mathrm{cm}}(t)\right],
$$

where $\boldsymbol{v}_{\mathrm{cm}}=\sum_{j}^{N_{c}} \boldsymbol{v}_{j} / N_{c}$ is the center-of-mass velocity of the $N_{c}$ particles in the cell. $\mathcal{R}(\alpha)$ is the rotation matrix. The orientation of the rotation axis is chosen anew in every collision step for every collision cell. The MPC algorithm, as described so far, lacks Galilean invariance. To restore Galilean invariance, a random shift of the collision grid is applied at any collision step as proposed in Ref. 33.

The dynamics of the $2 N_{m}$ monomers of mass $M$ each is determined by Newton's equations of motion

$$
M \ddot{\boldsymbol{r}}_{j}=\boldsymbol{F}_{j}, \quad j=1, \ldots, 2 N_{m}
$$

between the collisions, where $\boldsymbol{F}_{j}$ denotes the force acting on particle $j$ due to the potentials described in Sec. II. These equations are integrated by the velocity Verlet algorithm ${ }^{34}$ with the time step $h_{p}$.

Coupling of the solute and solvent dynamics is achieved by inclusion of the monomers in the collision step of the MPC algorithm. ${ }^{27,30,35}$ We only have to account for the larger masses $M$ of the monomers. Hence, the collision step is performed with a modified center of mass velocity $\boldsymbol{v}_{\mathrm{cm}}$ $=\left(\sum_{i}^{N_{c}} \boldsymbol{v}_{i}+\sum_{j}^{N_{M}} \boldsymbol{V}_{j} M / m\right) /\left(N_{c}+N_{M} M / m\right)$, where $\boldsymbol{V}_{j}$ is the velocity of monomer or counterion $j$, respectively, and $N_{M}$ is the number of such particles in the considered cell.

To keep the temperature constant, we scale the velocities of the particles for every cell independently at any collision step. ${ }^{36,37}$ This is necessary to remove the energy added to the system by the external electric field.

\section{PARAMETERS}

We measure lengths in units of $a$ and times in units of $\tau=\sqrt{m a^{2} / k_{B} T}$. The mean number of solvent particles in a collision cell is chosen as $\left\langle N_{c}\right\rangle=10$, the MPC collision time as $h=0.03 \tau$, and the MD time step as $h_{p}=10^{-2} \tau$. Furthermore, we consider a rotation angle of $\alpha=130^{\circ} \cdot{ }^{30,38}$ For the monomers we choose the mass $M=3 \mathrm{~m}$ and the diameter $\sigma$ 


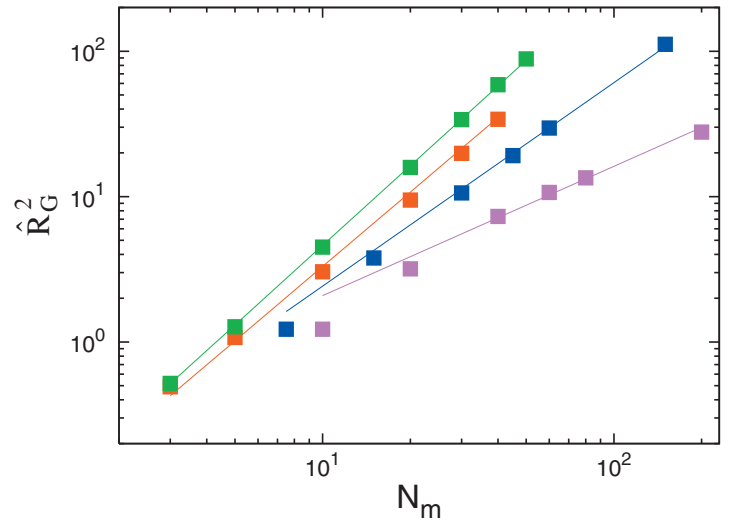

FIG. 1. Mean square radii of gyration $\hat{R}_{G}^{2}$ as function of the monomer number for the Coulomb interaction strengths $\hat{\gamma}=0.19$ (red), 1.9 (green), 5.7 (blue), and 9.5 (magenta). The data points for $\hat{\gamma}=5.7$ and 9.5 are shifted to the right by the factors 1.5 and 2 , respectively. The solid lines are fits to the data points, which yield the dependencies $\hat{R}_{G}^{2} \sim N_{m}^{2 \nu}$, with $\nu$ $\approx 0.85,0.91,0.7,0.45$ (top to bottom).

$=0.5 a$. This yields the mean bond length $l=0.53 a$, which is determined by Lennard-Jones repulsion of bound particles and the harmonic potential (1). The force constant is set to $\hat{\kappa}=10^{3}$ and $\epsilon / k_{B} T=1$. We present results for Coulomb interaction strengths $\hat{\gamma}=l_{B} / l=0.19,1.9,5.7,9.5$, with the Bjerrum length $l_{B}=e^{2} /\left(4 \pi \varepsilon \varepsilon_{0} k_{B} T\right)$, while the electric field strength $\hat{E}=E /\left(k_{B} T / a e\right)$ is fixed at the value $\hat{E}=0.1$. As has been shown in Refs. 23 and 39, this value is small enough to leave the polyelectrolyte conformations unperturbed, i.e., we are in the linear response regime. In order to compare results for various chain lengths, we keep the monomer density constant. Explicitly, for a chain of $N_{m}=10$ monomers, we choose the box length $L=22 a$.

\section{RESULTS}

\section{A. Polyelectrolyte conformations}

Figure 1 displays the polyelectrolyte radii of gyration for the various Coulomb interaction strengths. Evidently, they exhibit a severe dependence on $\hat{\gamma}$, as has been already demonstrated in Ref. 5. Over a certain range of monomer numbers, which depends on $\hat{\gamma}, \hat{R}_{g}^{2}$ is well described by a powerlaw, i.e., $\hat{R}_{g}^{2} \sim N_{m}^{2 \nu}$, with $\nu=\nu(\hat{\gamma})$. From the straight lines in Fig. 1 , we obtain the exponents $\nu(0.19) \approx 0.85, \nu(1.9)$ $\approx 0.91, \nu(5.7) \approx 0.7$, and $\nu(9.5) \approx 0.45$. The initial increase in the exponent with increasing interaction strength and decrease at larger $\hat{\gamma}$ is consistent with the results presented in Ref. 5. The increasing Coulomb interaction strength leads to a repulsion of the monomers. In the range of $\hat{\gamma} \approx 1$, counterion condensation sets in, which is accompanied by screening of the charge-charge interaction (see also Fig. 2). As a consequence, the radius of gyration decreases again. Very large interaction strengths even lead to an intramolecular attraction via the condensed counterions, which explains the $\nu$ value smaller than $1 / 2$, the value in a theta solvent. ${ }^{5}$ Ultimately, the value $\nu=1 / 3$ is obtained when all counterions are condensed. 5,11

The mean square end-to-end distances exhibit a very similar behavior as a function of chain length and interaction

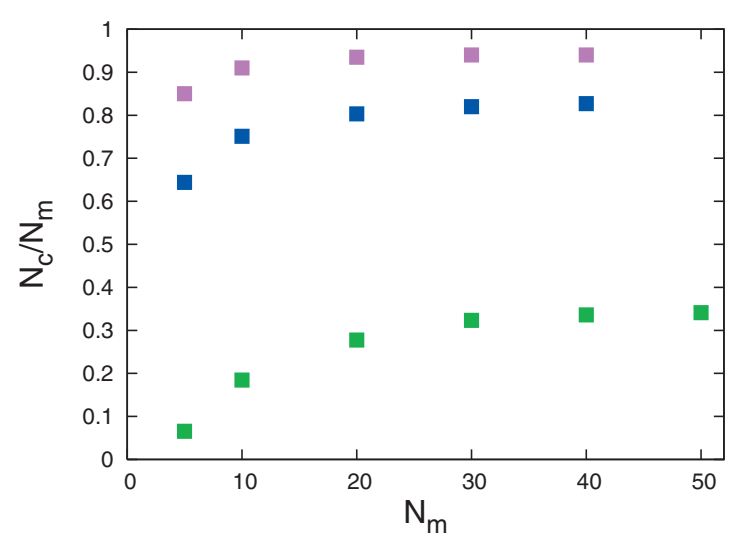

FIG. 2. Scaled number of condensed counterions $N_{c} / N_{m}$ of polyelectrolyte chains as function of the monomer number for the Coulomb interaction strengths $\hat{\gamma}=1.9,5.7$, and 9.5 (bottom to top).

strength, except that their exponents are somewhat larger. From the simulation data we find $\nu(0.19) \approx 0.9, \nu(1.9) \approx 1$, $\nu(5.7) \approx 0.7$, and $\nu(9.5) \approx 0.45$.

The differences in the scaling exponents of the radius of gyration and the mean square end-to-end distance is explained by the fact that a polyelectrolyte molecule is not self-similar. ${ }^{5}$ The charge-charge interactions induce new length scales, which destroy the scaling behavior valid for neutral linear macromolecules. 40,41

We like to emphasize that the considered polymers are flexible, but we observe rodlike scaling relations for $\hat{\gamma} \leqslant 2$ due to intramolecular Coulomb repulsion. This rodlike behavior must be understood in the sense of a blob model, i.e., a blob pole is formed, where the number of monomers per blob is $\gtrsim 1$. The obtained exponents are effective exponents for the considered interaction strengths and chain lengths, and characterize the system behavior in the crossover regime between different limiting scaling laws. A detailed discussion of scaling laws is presented in Ref. 42 .

\section{B. Counterion condensation}

The amount of condensed counterions depends strongly on the polymer length and interaction strength, as is shown in Fig. 2. (In addition, it also depends on polyelectrolyte concentration.) We consider a counterion as condensed, if it is within a distance of $2 \sigma$ from a monomer. ${ }^{5,23}$ Other measures to determine the effective charge have been suggested, ${ }^{39,43,44}$ but the differences are typically negligible. For all chain lengths, $N_{c} / N_{m}$ is approximately zero at $\hat{\gamma}$ $=0.19$. This changes when the Coulomb interaction strength increases. Then, the number of condensed counterions increases, which is simply due to a stronger attraction among oppositely charged ions. The chain-length dependence of $N_{c} / N_{m}$ is related to the finite size of the polymers. Longer polymers correspond to larger spatial charge densities which lead to a stronger attraction of the counterions. The same behavior can be observed for polyelectrolytes at equilibrium. ${ }^{5,6,10,11,45}$ 


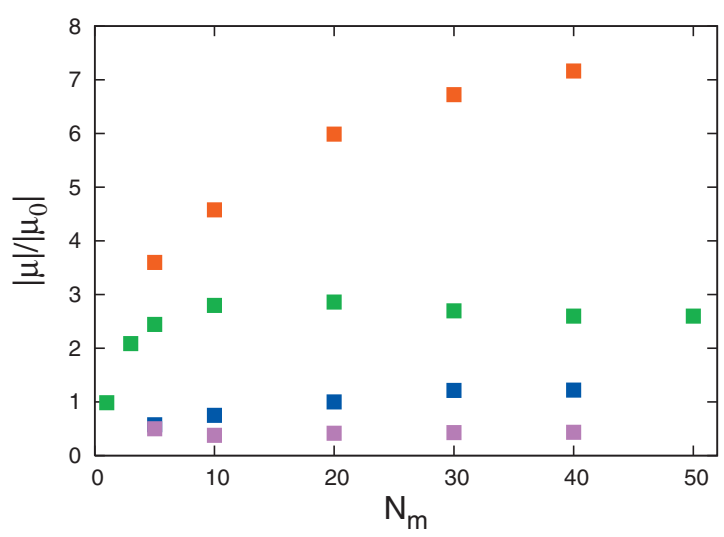

FIG. 3. Electrophoretic mobilities $\mu / \mu_{0}$ of polyelectrolyte chains as function of the monomer number $N_{m}$ and Coulomb interaction strengths $\hat{\gamma}$ $=0.19,1.9,5.7$, and 9.5 (top to bottom).

\section{Electrophoretic mobility}

Figure 3 shows mobilities of polyelectrolytes at various Coulomb interaction strengths and $\hat{E}=0.1$ as function of the monomer number $N_{m}$. We measure $\mu$ in units of the bare mobility $\mu_{0}$, which we determine from a simulation of a single monomer in presence of the solvent. The mobilities of the polyelectrolyte chains decrease with increasing Coulomb interaction strength for two reasons: On the one hand, counterion condensation reduces the actual charge of the polyelectrolyte. On the other hand, screening of the Coulomb interaction among the monomers by counterions (condensed and free) leads to a chain-length independent mobility. ${ }^{13,14}$ More details on charge screening will be presented in an upcoming article. ${ }^{46}$

Naturally, an increasing Coulomb interaction strength also affects the behavior of the condensed and the uncondensed counterions. Their mobilities are shown in Fig. 4 for the various Coulomb interaction strengths. For the uncondensed counterions we present error bars since the fluctuations in their mobilities become large with increasing $\hat{\gamma}$. Error bars for the polyelectrolyte chains and the condensed counterions are not depicted. Here, the uncertainties are between $5 \%$ and $10 \%$.

We will start by discussing the case $\hat{\gamma}=0.19$ for which the mobilities are represented by Fig. 4(a) (and Fig. 3 for the polyelectrolytes only). The electrostatic interaction between the counterions and the polyelectrolyte is very weak over the considered range of chain lengths. Hence, here, the multicomponent system (polyelectrolytes and counterions) decouples into two almost independent subsystems. As a consequence, the counterion mobilities are equal to the bare mobility of a single ion. At the same time, the polyelectrolyte behaves almost as an uncharged polymer in sedimentation. Thus, the Nernst-Einstein relation $\mu=D Q /\left(k_{B} T\right)$ applies approximately, where $Q$ is the total charge of the polymer and $D$ its diffusion coefficient. ${ }^{24,47}$ Since the polyelectrolyte exhibits close to rodlike conformations (see Fig. 1), we expect a logarithmic increase in its mobility due to hydrodynamic interactions, i.e., $\mu \sim \ln \left(N_{m}-1\right),{ }^{14,40,48}$ which is indeed confirmed by the simulations [cf. Fig. 4(a) and Sec. V D].

For $\hat{\gamma}=1.9$, the counterion and polyelectrolyte dynamics
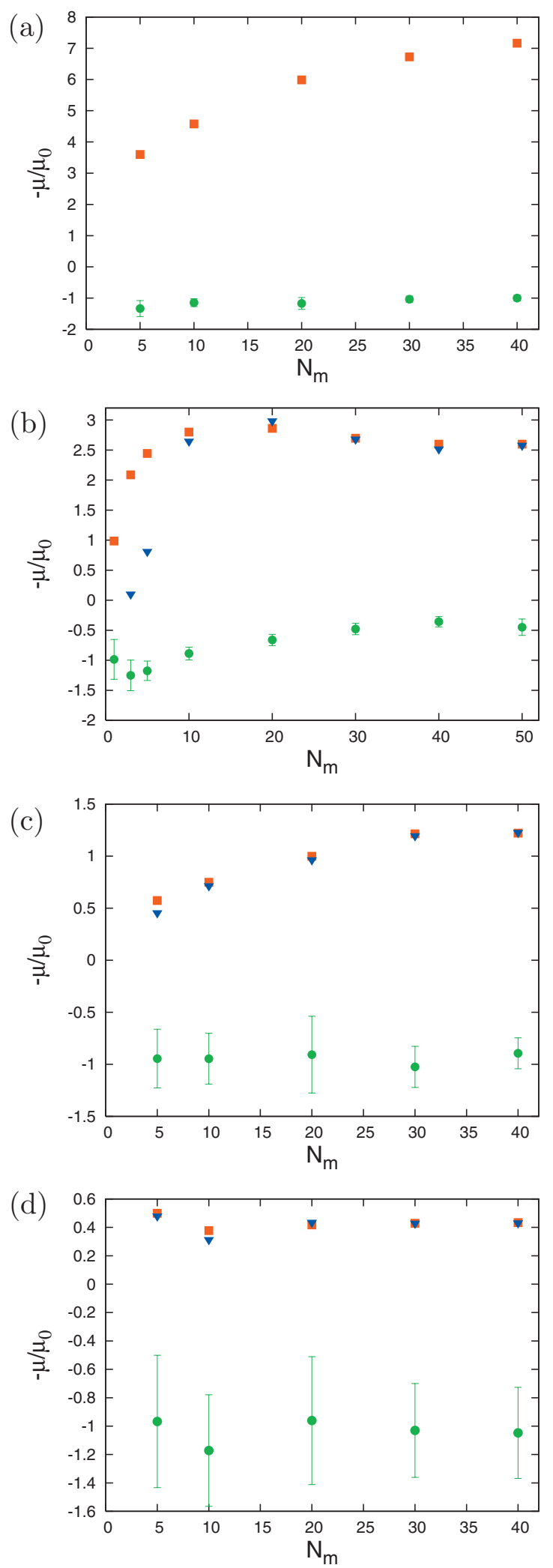

FIG. 4. Electrophoretic mobilities $\mu / \mu_{0}$ of polyelectrolyte chains (squares), condensed (triangles), and uncondensed (bullets) counterions as function of the monomer number $N_{m}$ for the Coulomb interaction strengths (a) $\hat{\gamma}$ $=0.19$, (b) 1.9 , (c) 5.7 , and (d) 9.5 .

are strongly coupled as already discussed in Ref. 23. Here, the multicomponent character of the solution is reflected in the mobility and the Nernst-Einstein relation does not apply. The initial increase in the chain mobility is due to hydrody- 


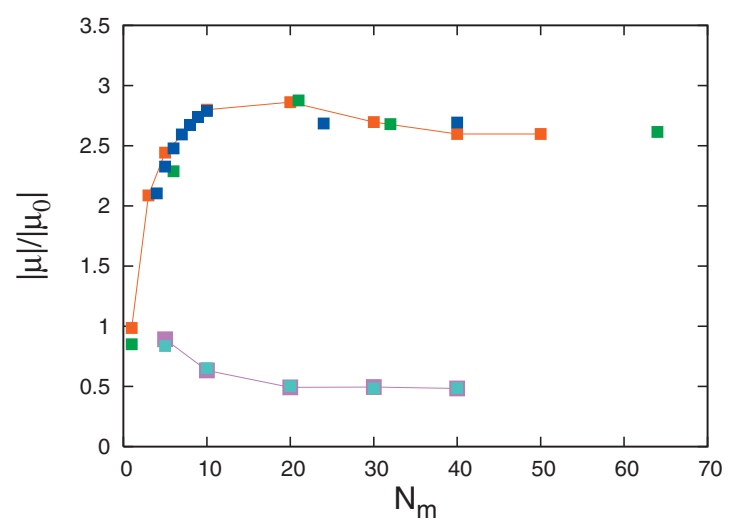

FIG. 5. Electrophoretic mobilities $\mu / \mu_{0}$ of polyelectrolyte chains as function of the monomer number for $\hat{\gamma}=1.9$ with (red squares) and without (magenta squares) hydrodynamic interactions. (Lines are guides for the eye only.) The light blue squares correspond to the dependence $\mu / \mu_{0}=\left(N_{m}\right.$ $\left.-N_{c}\right) /\left(N_{m}+N_{c}\right)$. Blue and green squares indicate experimental results on PSS obtained by capillary electrophoresis (Ref. 16) and NMR (Refs. 18 and $22)$, respectively.

namic interactions. For $N_{m} \geqslant 10$, counterion condensation and charge screening ${ }^{49}$ lead to a decrease in the mobility. ${ }^{22,23}$ The parameter $\hat{\gamma}=1.9$ closely corresponds to interaction strengths of short PSS molecules and we find a quantitative agreement of the mobilities with experimental results from capillary electrophoresis ${ }^{16}$ and $\mathrm{NMR}^{18,50}$ as shown in Fig. $5 .^{23}$ The comparison of the simulation results with and without hydrodynamic interactions clearly reveals that the initial increase in the polyelectrolyte mobility is caused by hydrodynamic interactions.

Evidently, the condensed counterions move with the polyelectrolyte for $N_{m}>10$. For short molecules, condensed counterions exhibit a smaller mobility than the polyelectrolyte. At the same time, uncondensed ions exhibit a mobility similar to that of a single free ion within the accuracy of the simulation. The condensed ions are only very weakly attached to the polyelectrolyte, and slide slowly along the molecule in the direction opposite to its velocity (in the reference frame of the molecule), thereby moving in the same direction as the molecule. For longer molecules, the ion mobilities are by a factor of two smaller than $\mu_{0}$. The net charge of the counterion-polyelectrolyte complex is sufficiently large to attract the uncondensed ions and decrease their mobility. We like to point out that in our reference frame the total momentum of all particles, i.e., polymer, counterions, and fluid, is zero.

At the interaction strength $\hat{\gamma}=5.7$ [see Fig. 4(c)], condensed counterions move with the polyelectrolyte and the mobilities of the free ions are equal to $\mu_{0}$. The net charge of the complex is too small to affect the uncondensed ions. The mobility of the polyelectrolyte increases with increasing molecular weight for small monomer numbers and seems to approach a length-independent value for larger $N_{m}$.

For even larger interaction strengths, the polyelectrolyte mobility is almost independent of molecular weight [see Fig. 4(d)], and the magnitude of the free counterion mobility is again equal to $\mu_{0}$.

As pointed out before, we attribute the chain-length independence of the polyelectrolyte mobility for the interaction

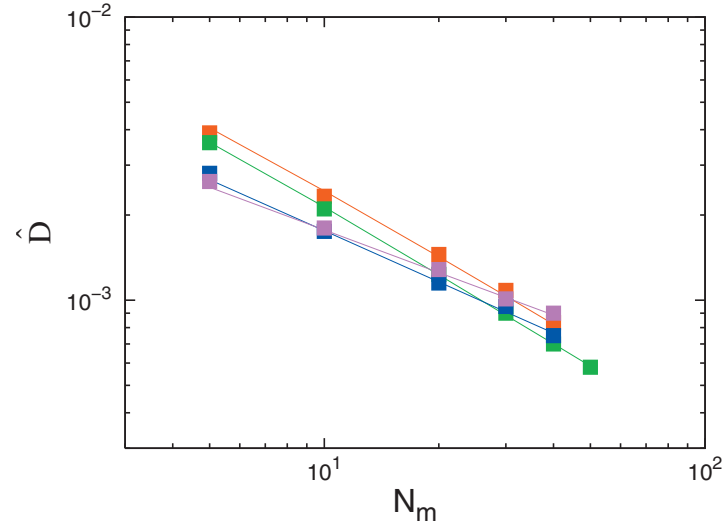

FIG. 6. Diffusion coefficients $\hat{D}$ as function of monomer number $N_{m}$ for the Coulomb interaction strengths $\hat{\gamma}=0.19$ (red), 1.9 (green), 5.7 (blue), 9.5 (magenta). The lines for $\hat{\gamma}=0.19$ and $\hat{\gamma}=1.9$ are calculated according to Eqs. (8) and (9), respectively (see Ref. 23). The lines for $\hat{\gamma}=5.7,9.5$ are powerlaw fits which exhibit the scaling behavior $D \sim N_{m}^{-0.6}$ and $D \sim N_{m}^{-0.5}$, respectively.

strengths $\hat{\gamma}=1.9,5.7$, and 9.5 at large $N_{m}$ to the screening of the charges by the condensed counterions. At $\hat{\gamma}=9.5$, the net charge is so small that an efficient screening is already achieved for short polymers.

\section{Polyelectrolyte diffusion coefficient}

As pointed out before, we consider a multicomponent system composed of polymer, counterions, and fluid. To unravel the influence of the individual components on the polyelectrolyte mobility, we will study the polyelectrolyte diffusion coefficients for the various interaction strengths. This will provide insight into the relevance of hydrodynamic interactions in electrophoresis. We will present results for equilibrium simulations only, but we like to point out that the calculation of diffusion coefficients in the presence of the electric field perpendicular to its direction yields the same values.

Because of the rodlike nature of the polyelectrolyte at $\hat{\gamma}=0.19$, we expect that the chain-length dependence of its diffusion coefficient can be described by the expression for rodlike objects in the presence of hydrodynamic interactions. Figure 6 displays diffusion coefficients for polymers of various lengths and interaction strengths, which are determined via the center-of-mass mean square displacements. The data for $\hat{\gamma}=0.19$ are well described by the expression $23,40,48,51,52$

$$
\hat{D}=\frac{a}{3 \pi \hat{\nu} \rho l}\left(\frac{\delta_{1}}{N_{m}}+\frac{\delta_{2}}{N_{m}-1}\left[\ln \left(N_{m}-1\right)-0.21\right]\right)
$$

valid for rodlike objects, where local friction as well as hydrodynamic interactions are taken into account. In dimensionless units the diffusion coefficient is given by $\hat{D}$ $=\tau D / a^{2}$, and the kinematic viscosity of our fluid is $\hat{\nu}$ $=\tau \nu / a^{2}=2.75$. The parameters $\delta_{1}$ and $\delta_{2}$ are $\delta_{1}=1.1$ and $\delta_{2}$ $=0.95 . \delta_{1}$ is larger than unity since the discrete time dynamics of the solvent leads to diffusion coefficients which can be quantitatively different from those given by a Langevin dynamics, depending on the parameters of the MPC algorithm. $\delta_{2}$ is smaller than unity simply because the observed confor- 
mations of the chain are not perfectly rodlike. They behave in a rodlike manner only. In Eq. (8), counterion condensation is completely neglected, in agreement with the data displayed in Fig. 2.

The Nernst-Einstein relation implies that $\hat{\mu} /(\hat{D} \hat{Q})=1$. From the numerical data, an almost chain-length independent ratio $\hat{\mu} /(\hat{D} \hat{Q})=0.84 \pm 0.1$ is obtained. Thus, the NernstEinstein relation is approximately satisfied, as expected.

For the larger interaction strength $\hat{\gamma}=1.9$, condensed counterions modify the diffusion coefficient. The data of Fig. 6 are well described by a similar expression to Eq. (8), but now the frictional contribution of the condensed counterions has to be taken into account, which gives

$$
\hat{D}=\frac{a}{3 \pi \hat{\nu} \rho l}\left(\frac{\delta_{1}}{N_{m}+N_{c}}+\frac{\delta_{2}}{N_{m}-1}\left[\ln \left(N_{m}-1\right)-0.19\right]\right),
$$

with $\delta_{1}=1.37$ and $\delta_{2}=0.79$. A more detailed discussion of the results for this interaction strength can be found in Ref. 23, where we also present a comparison with experimental results obtained by NMR. ${ }^{18,53,54}$ Note that the values 0.21 and 0.19 in Eqs. (8) and (9) are fit parameters, which include the effective thickness of the particles. Since we consider point particles, their effective thickness is determined by the MPC algorithm and is on the order of the bond length.

The diffusion coefficients for the large interaction strengths are not expected to exhibit a logarithmic chainlength dependence anymore because for these $\hat{\gamma}$, the radii of gyration show power-law dependencies, which correspond rather to polymers in good solvent or theta solvent, respectively, than to a rod. Thus, we fit the data by a power-law $\hat{D} \sim N_{M}^{-\alpha}$, which yields the exponents $\alpha=0.6$ for $\hat{\gamma}=5.7$ and $\alpha=0.5$ for $\hat{\gamma}=9.5$. These exponents are in good agreement with the expectation $\hat{D} \sim \hat{R}_{G}^{-1}$. However, this relation applies to long polymers only. The short-time center-of-mass diffusion coefficient in the over damped regime described by the Smoluchowski equation ${ }^{40}$ of a polymer is given by the Kirkwood formula ${ }^{30,55-57}$

$$
D=\frac{D_{0}}{N_{m}}+\frac{k_{B} T}{6 \pi \eta} \frac{1}{R_{H}}
$$

where $D_{0}$ is the friction coefficient of a single monomer and $R_{H}$ is the hydrodynamic radius. For long polymers $R_{H} \sim R_{G}$ and $D_{H}=k_{B} T /\left(6 \pi \eta R_{H}\right)$ dominates over $D_{0} / N_{m}$, hence, $D$ $\sim R_{G}^{-1}$ for such molecular weights. The considered polymer lengths are too short to follow the dependence $D \sim D_{H}$, thus, the observed scaling of the diffusion coefficients for large $\hat{\gamma}$ with respect to the radius of gyration is just a coincidence. The comparison of $D_{H}$, where hydrodynamic radii are taken from the simulations, with $D-D_{0} /\left(N_{m}+N_{c}\right)$ shows good agreement within the accuracy of the simulations.

The Nernst-Einstein relation is not satisfied for the discussed interaction strengths $\hat{\gamma} \geq 1.9$ due to the multicomponent nature of the solution.

The comparison of the simulation results with theoretical expectations clearly reveals that the diffusion coefficients are determined by hydrodynamic interactions. For none of the considered interaction strengths are hydrodynamic interactions screened.

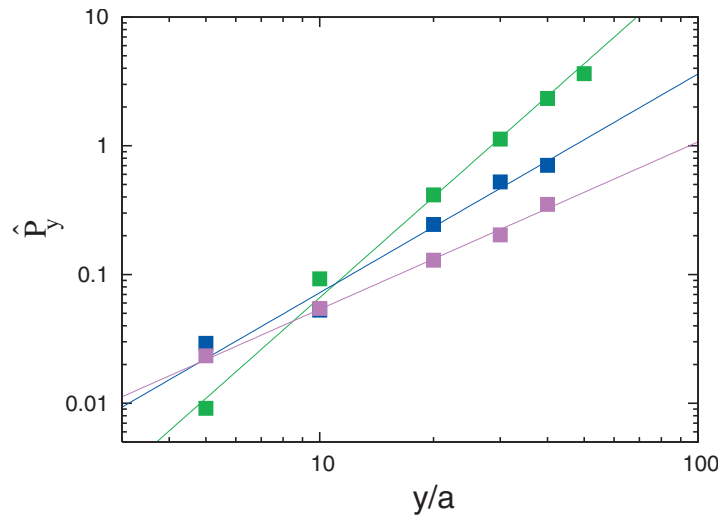

FIG. 7. Dipole moment $\hat{P}_{y}$ of a polyelectrolyte as function of the monomer number $N_{m}$ for the Coulomb interaction strengths 1.9 (green), 5.7 (blue), and 9.5 (magenta).

\section{E. Polarization of the polyelectrolyte-counterion complex}

The polyelectrolyte-counterion complex can be polarized by the applied electric field. To characterize the extent of polarization, we calculate the dipole moment $\hat{P}_{y}$ along the orientation of the electric field according to

$$
\hat{P}_{y}=\sum_{i} \hat{q}^{p}\left(\hat{r}_{i y}^{p}-\hat{r}_{\mathrm{cm}, y}^{p}\right)+\sum_{j} \hat{q}^{c}\left(\hat{r}_{i y}^{c}-\hat{r}_{\mathrm{cm}, y}^{p}\right),
$$

where $\hat{q}^{p}=-1$ is the charge of a monomer and $\hat{q}^{c}=1$ is the charge of a counterion. $\hat{r}_{\mathrm{cm}, y}^{p}$ denotes the position of the center-of-mass of the polyelectrolyte. Hence, the first term is a summation over the chain monomers, and the second term accounts for condensed counterions. In dimensionless units the dipole moment is given by $\hat{P}=P /(e a)$. The polarization $\alpha_{p}$ is defined as $\alpha_{p}=P / E$.

Since there are no counterions condensed for $\hat{\gamma}=0.19$, the simulations yield no polarization for that system. The dipole moments for the other interaction strengths are displayed in Fig. 7. The dependence of $\hat{P}_{y}$ on the monomer number is well described by the power-law $\hat{P}_{y} \sim N^{\nu^{\prime}}$, with $\nu^{\prime}=2.6,1.7$, and 1.3, respectively, for $\hat{\gamma}=1.9,5.7$, and 9.5.

The polarization of a homogeneous, spherical charge distribution of radius $R$ and total charge $Q$ around a point charge $Q^{\prime}$ is $\alpha_{p} \sim Q R^{3} / Q^{\prime}{ }^{11,58}$ Since our polyelectrolytecounterion complex has no preferred orientation, it should, on the average, resemble a spherical object and, hence, we replace $R$ by the polyelectrolyte radius of gyration. The charge $Q$ is proportional to the amount of condensed counterions and $Q^{\prime}$ to the number of monomers. Thus, we expect a scaling behavior $\hat{\alpha}_{p} \sim N_{m}^{3 \nu}$ (cf. Sec. V A) for a constant ratio $N_{m} / N_{c}$, which is approximately satisfied for $N_{m} \geq 20$. With the $\nu$ values from Fig. 1 , we find $3 \nu \approx 2.7,2.1,1.4$, respectively. Hence, the simulation results approximately follow the expected behavior.

In Fig. 8, we additionally show the distributions $P_{c}\left(r_{y}\right)$ of the counterions along the orientation of the electric field for the various chain lengths and Coulomb interaction strengths. Here, $r_{y}$ is the distance from the center-of-mass of a polyelectrolyte chain. The probability distribution $P_{c}$ is normalized such that its integral over the length of the simu- 

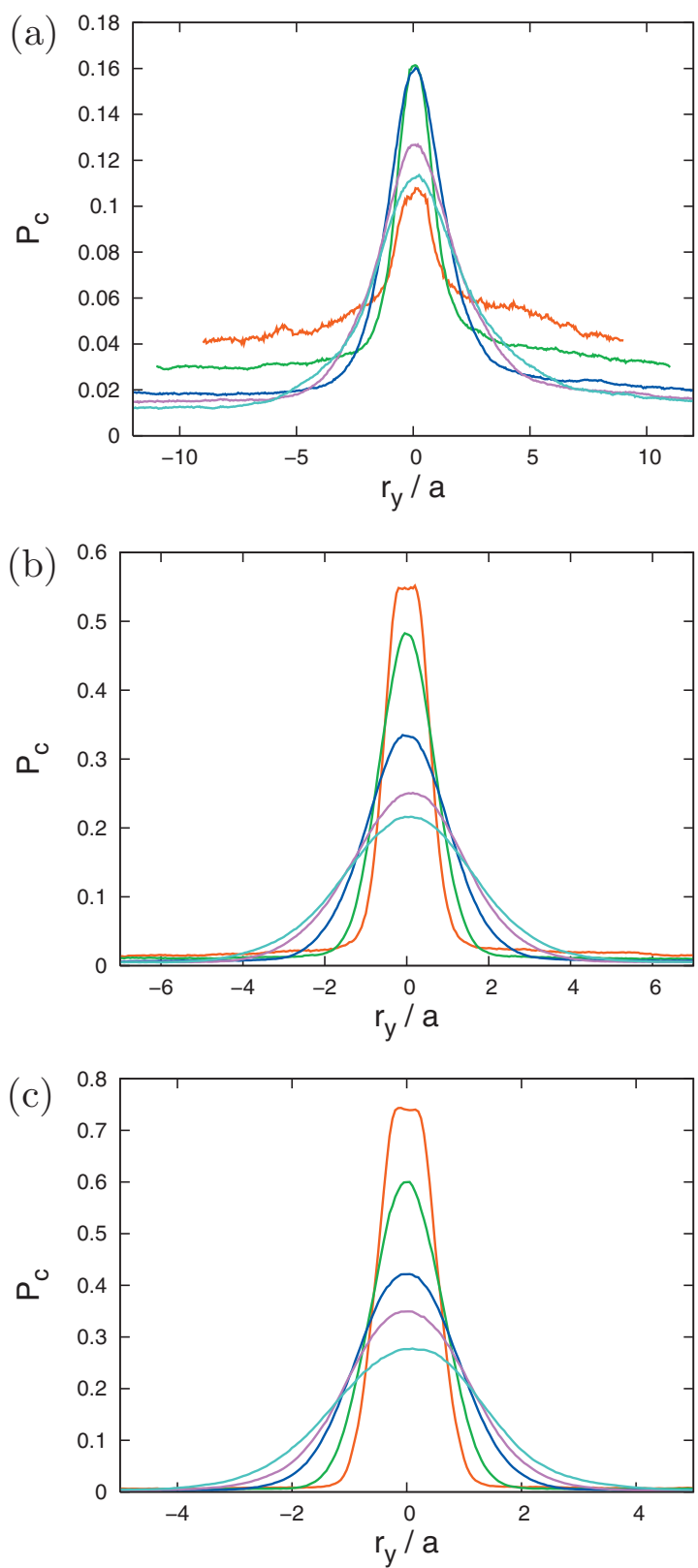

FIG. 8. Counterion distributions as function of the distance $\hat{r}_{y}$ from the center-of-mass of a polyelectrolyte chain along the direction of the electric field. The Coulomb interaction strengths are (a) $\hat{\gamma}=1.9$, (b) 5.7, and (c) 9.5 . Within each plot, results are shown for the chain lengths $N_{m}=5$ (red), 10 (green), 20 (blue), 30 (magenta), and 40 (light blue).

lation cell is unity. The number of monomers at a particular $r_{y}$ is obtained by multiplying the distribution with the number of monomers per molecule.

The distributions of ions for $\hat{\gamma}=0.19$ are homogeneous for all considered chain lengths and therefore are not shown. With the onset of counterion condensation, the distribution functions develop a peak close to the center-of-mass of the polymer. Since only a fraction of ions is condensed for $\hat{\gamma}$ $=1.9$, the distribution functions do not decay to zero, they rather approach a constant value at large distances corresponding to the concentration of uncondensed ions, as shown in Fig. 8(a). Although the electric field is weak, it induces a charge asymmetry, with more ions at $r_{y}>0$, which is reflected in the dipole moment of Fig. 7. The increase in the probability distribution at $r_{y}=0$, when the chain length is increased from $N_{m}=5$ to $N_{m}=10$, reflects the increase in the amount of condensed counterions for the longer polymer. The larger amount of condensed ions for $N_{m}=20$ leads to a broadening of the ion distribution, which is even more pronounced for longer chains. The distributions for $N_{m}=40$ and $N_{m}=50$ are rather similar due to the saturation of ion condensation.

At larger interaction strengths, more ions are condensed even at short polymers and the distributions are narrower at the same polymer length. The distributions still assume finite values for $\hat{\gamma}=5.7$ far away from the center-of-mass [Fig. 8(b)], whereas for $\hat{\gamma}=9.5 P_{c}$ essentially decays to zero [Fig. 8(c)]. At the same time, the extent of asymmetry decreases with increasing interaction strength and is only present for the longer polymers, i.e., for $N_{m}=40$ and $N_{m}=50$. For chain lengths $N_{m} \geqslant 10$, the distribution functions in the vicinity of the center-of-mass, i.e., for small $r_{y}$, can be well fitted by Gaussian functions, but the tails decay much slower and therefore reach far out into the solvent.

\section{CONCLUSIONS}

We have studied the structural, dynamical, and transport properties of polyelectrolytes in solution which are exposed to a weak electric field by mesoscale hydrodynamic simulations. Various polymer lengths and Coulomb interaction strengths have been considered. Moreover, we have addressed the importance of hydrodynamic interactions on the dynamics of charged polymers.

Our studies of the diffusion of the polyelectrolytes in the presence of the electric field reveal that hydrodynamic interactions are present at any Coulomb interaction strength. At weak Coulomb interactions, the polymer conformations are rodlike and hence the diffusion coefficient exhibits a logarithmic dependence on molecular weight. At larger Coulomb interactions, longer chains exhibit coiled conformations and the diffusion coefficient can be described within the Zimm model. ${ }^{30,40,55}$ The cross-over from a rodlike to a coil-like behavior occurs within a narrow range of Coulomb interaction strengths, namely, between $2 \lessgtr \hat{\gamma} \leqslant 5$ in our simulations. This is related to the strong conformational changes of the polymers by counterion condensation within this range of interactions. ${ }^{5,10}$ This also explains the fact that the polymer length dependence of diffusion coefficients changes from a logarithmic dependence to a power-law behavior.

Hydrodynamic interactions cause an initial increase in the electrophoretic mobility at small chain lengths and interaction strengths $\hat{\gamma}<5$. Here, the range of chain lengths depends on $\hat{\gamma}$ because counterion condensation sets in at higher molecular weights for smaller interaction strengths. At large molecular weight, the mobility becomes independent of chain length due to counterion condensation and screening of intramolecular charge-charge interactions by the counterion cloud.

Our studies demonstrate the relevance of the multicomponent nature-polyelectrolyte, counterions, salt, and solvent — of a polyelectrolyte solution on its dynamics and transport properties in an electric field. For weak interaction 
strengths and shorter chain lengths, the Nernst-Einstein relation applies. ${ }^{23,39}$ Here, solvent-mediated interactions are important. When counterion condensation sets in, the relation is violated, ${ }^{23,24}$ which we explain by a screening of the intramolecular Coulomb interactions by ions, i.e., counterions or salt ions dominated the transport properties in the field. Currently, further studies are underway to achieve a better understanding of the influence of charge screening on the electrophoretic mobility.

${ }^{1}$ M. Schmidt, Polyelectrolytes with Defined Molecular Architecture I and II, Advances in Polymer Physics Vol. 165-166 (Springer, Berlin, 2004).

${ }^{2}$ M. Stevens and K. Kremer, Phys. Rev. Lett. 71, 2228 (1993).

${ }^{3}$ M. O. de la Cruz, L. Belloni, M. Delsanti, J. P. D. O. Spalla, and M. Drifford, J. Chem. Phys. 103, 5781 (1995).

${ }^{4}$ V. A. Bloomfield, Biopolymers 44, 269 (1997).

${ }^{5}$ R. G. Winkler, M. Gold, and P. Reineker, Phys. Rev. Lett. 80, 3731 (1998).

${ }^{6}$ G. S. Manning and J. Ray, J. Biomol. Struct. Dyn. 16, 461 (1998).

${ }^{7}$ E. Raspaud, M. O. de la Cruz, J.-L. Sikorav, and F. Livolant, Biophys. J. 74, 381 (1998).

${ }^{8}$ M. O. Khan and B. Jönsson, Biopolymers 49, 121 (1999).

${ }^{9}$ Y. Yamasaki, Y. Teramoto, and K. Yosjikawa, Biophys. J. 80, 2823 (2001).

${ }^{10}$ S. Liu and M. Muthukumar, J. Chem. Phys. 116, 9975 (2002).

${ }^{11}$ R. R. Netz, Phys. Rev. Lett. 90, 128104 (2003).

${ }^{12}$ P. M. Résibois, Electrolyte Theory (Harper \& Row, New York, 1968).

${ }^{13}$ G. S. Manning, J. Phys. Chem. 85, 1506 (1981).

${ }^{14}$ M. Muthukumar, J. Chem. Phys. 107, 2619 (1997).

${ }^{15}$ D. A. Hoagland, E. Arvanitidou, and C. Welch, Macromolecules 32, 6180 (1999).

${ }^{16}$ H. Cottet, P. Gareil, O. Theodoly, and C. E. Williams, Electrophoresis 21, 3529 (2000).

${ }^{17}$ E. Stellwagen and N. C. Stellwagen, Electrophoresis 23, 2794 (2002).

${ }^{18}$ U. Böhme and U. Scheler, Macromol. Chem. Phys. 208, 2254 (2007).

${ }^{19}$ A. R. Völkel and J. Noolandi, J. Chem. Phys. 102, 5506 (1995).

${ }^{20}$ J.-L. Barrat and J.-F. Joanny, Adv. Chem. Phys. 94, 1 (1996).

${ }^{21}$ J. L. Viovy, Rev. Mod. Phys. 72, 813 (2000).

${ }^{22}$ K. Grass, U. Böhme, U. Scheler, H. Cottet, and C. Holm, Phys. Rev. Lett. 100, 096104 (2008).

${ }^{23}$ S. Frank and R. G. Winkler, EPL 83, 38004 (2008).

${ }^{24}$ E. Stellwagen, Y. Lu, and N. C. Stellwagen, Biochemistry 42, 11745 (2003).

${ }^{25}$ A. Malevanets and R. Kapral, J. Chem. Phys. 110, 8605 (1999).

${ }^{26}$ A. Malevanets and R. Kapral, J. Chem. Phys. 112, 7260 (2000).

${ }^{27}$ M. Ripoll, K. Mussawisade, R. G. Winkler, and G. Gompper, Europhys. Lett. 68, 106 (2004).
${ }^{28}$ R. Kapral, Adv. Phys. 140, 89 (2008).

${ }^{29}$ G. Gompper, T. Ihle, D. M. Kroll, and R. G. Winkler, Adv. Polym. Sci. 221, 1 (2009)

${ }^{30}$ K. Mussawisade, M. Ripoll, R. G. Winkler, and G. Gompper, J. Chem. Phys. 123, 144905 (2005).

${ }^{31}$ P. Ewald, Ann. Phys. 369, 253 (1921).

${ }^{32}$ B. Dünweg and A. J. C. Ladd, Adv. Polym. Sci. 221, 89 (2009).

${ }^{33}$ T. Ihle and D. M. Kroll, Phys. Rev. E 63, 020201(R) (2001).

${ }^{34}$ W. C. Swope, H. C. Andersen, P. H. Berens, and K. R. Wilson, J. Chem. Phys. 76, 637 (1982).

${ }^{35}$ A. Malevanets and J. M. Yeomans, Europhys. Lett. 52, 231 (2000).

${ }^{36}$ M. P. Allen and D. J. Tildesley, Computer Simulation of Liquids (Clarendon, Oxford, 1987).

${ }^{37}$ C.-C. Huang, A. Chatterji, G. Sutmann, G. Gompper, and R. G. Winkler, J. Comput. Phys. 229, 168 (2010).

${ }^{38}$ M. Ripoll, K. Mussawisade, R. G. Winkler, and G. Gompper, Phys. Rev. E 72, 016701 (2005).

${ }^{39}$ K. Grass and C. Holm, Soft Matter 5, 2079 (2009).

${ }^{40}$ M. Doi and S. F. Edwards, The Theory of Polymer Dynamics (Oxford University Press, New York, 1986).

${ }^{41}$ P.-G. de Gennes, Scaling Concepts in Polymer Physics (Cornell University Press, Ithaca, 1979).

${ }^{42}$ H. Schiessel and P. Pincus, Macromolecules 31, 7953 (1998).

${ }^{43}$ L. Belloni, M. Drifford, and P. Turq, Chem. Phys. 147, 83 (1984).

${ }^{44}$ M. Deserno, C. Holm, and S. May, Macromolecules 33, 199 (2000).

${ }^{45}$ R. R. Netz, J. Phys. Chem. B 107, 8208 (2003).

${ }^{46}$ R. G. Winkler and S. Frank (to be published).

${ }^{47}$ A. E. Nkodo, J. M. Garnier, B. Tinland, H. Ren, C. Desruisseaux, L. C. McCormick, G. Drouin, and G. W. Slater, Electrophoresis 22, 2424 (2001).

${ }^{48}$ M. M. Tirado, C. L. Martínez, and J. G. de la Torre, J. Chem. Phys. 81, 2047 (1984).

${ }^{49}$ T. Hofmann, R. G. Winkler, and P. Reineker, J. Chem. Phys. 118, 6624 (2003).

${ }^{50}$ U. Scheler, in Handbook of Polyelectrolytes and Their Applications, edited by S. K. Tripaty, J. Kumar, and H. S. Nalwa (American Scientific, Los Angeles, 2002), p. 173.

${ }^{51}$ R. G. Winkler, K. Mussawisade, M. Ripoll, and G. Gompper, J. Phys.: Condens. Matter 16, S3941 (2004).

${ }^{52}$ R. G. Winkler, J. Chem. Phys. 127, 054904 (2007).

${ }^{53}$ U. Böhme and U. Scheler, Colloids Surf., A 222, 35 (2003).

${ }^{54}$ U. Böhme and U. Scheler, J. Colloid Interface Sci. 309, 231 (2007).

${ }^{55}$ L. Harnau, R. G. Winkler, and P. Reineker, J. Chem. Phys. 104, 6355 (1996).

${ }^{56}$ J. G. Kirkwood and J. Riseman, J. Chem. Phys. 16, 565 (1948).

${ }^{57}$ J. P. Erpenbeck and J. G. Kirkwood, J. Chem. Phys. 29, 909 (1958).

${ }^{58}$ Theory of Electric Polarization, edited by C. J. F. Böttcher (Elsevier, Amsterdam, 1973). 